\title{
It is the very model of a modern model organism
}

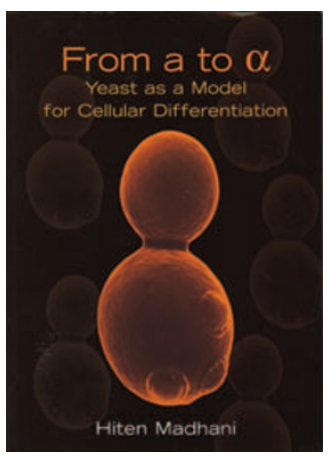

\section{From a to $\alpha$ : Yeast as a Model for Cellular Differentiation}

\author{
By Hiten Madhani \\ Cold Spring Harbor Laboratory Press • \\ 2007 \\ $£ 35 / \$ 59$ (Hardcover) \\ $£ 22$ / \$39 (Paperback)
}

\section{Jeremy Thorner}

In the 1970 edition of his textbook, Molecular Biology of the Gene, Jim Watson (a modern major-general of biology, by any measure) made a prescient remark. He said there were many reasons to allow a nucleated microbe to share the research limelight with phage and Escherichia coli. Selecting a tractable model was one approach to study features that define eukaryotic cells and the logical choice was baker's yeast, Saccharomyces cerevisiae. A concise new book by Hiten Madhani vindicates Watson's perspicacity. General principles, common themes and molecular mechanisms that apply as much to humans as to fungi, were first recognized in this organism. Thus, studies in S. cerevisiae have had the pioneering role for geneticists, biochemists, molecular biologists and cell biologists that Watson envisioned. Madhani's book thoughtfully highlights the elegant and ground-breaking work performed in yeast, and the resulting general mechanistic insights and molecular paradigms that emerged.

In the preface, Madhani states the book's mission - to tell the 'story' of what has been learned from yeast about hallmark processes in cellular differentiation. The first two chapters introduce yeast biology, including the $S$. cerevisiae life cycle and its three cell types, and draw analogies to human gametogenesis and fertilization. Other topics covered include the mating process, responses to nutrient limitation and the cell cycle and budding pattern (chapter 8 expands on this last topic). Chapter 3 focuses on the genetic loci that function as the master regulators of cell type, on the DNA-binding transcription factors that they encode, the cis-acting sequences at which they act, the coactivators and corepressors with which they interact and the gene-regulatory schemes that control cell type-specific gene expression that result from such combinatorial flexibility. The last chapter presents variations on this theme by discussing mating-type determination in two human pathogens, Cryptococcus neoformans and Candida albicans. Chapters 4 and 5 highlight the insights gained about peptide hormone biogenesis and secretion, G-protein-coupled receptors, MAPK cascades and other principles of signal transduction - all first gleaned from studying the production of and response to yeast mating pheromones. Chapter 6 discusses homothallism (mating-type switching) and its parallels to stem-cell renewal, unequal cell division and compartmentalization of

Jeremy Thorner is in the Department of Molecular and Cell Biology at the University of California at Berkeley, Room 16, Barker Hall, Berkeley, CA 94720-3202, USA.

e-mail: jthorner@berkeley.edu transcription factors. Chapter 7 spotlights silencing of the latent telomeric mating-type loci and what these studies have taught us about the establishment and spreading of heterochromatin, histone modification, and the effects of epigenetic marking on gene expression and accessibility for recombination.

In the specific sense that Madhani intended - as a manual for how yeast studies have led to general principles of eukaryotic cell differentiation - the book succeeds admirably, and proceeds in a logical, iterative and readable manner. It benefits from simple graphics and inserts in most chapters, which present specific concepts in the context of metazoans or of human disease. However, there are limitations: for certain subjects, important nuances get sacrificed at the altar of clarity. For example: Gpa1 (heterotrimeric $G$ protein a subunit) is shown interacting with the cytosolic tail of the pheromone receptor, but its primary contacts are actually with the third intracellular loop; Ste5 is shown associating with the plasma membrane solely by tethering to membraneanchored Ste4-Ste18 (G $\beta \gamma$ complex), but an internal PH domain and an amino-terminal amphipathic a-helix are also essential; and so on. There are also unfortunate typographical and even some factual errors - the pheromone precursor encoded by $M F \alpha 2$ is said to contain six a-factor repeats, but in fact, it has only two; Ste14 is shown making a proteolytic cleavage near the N-terminus of the a-factor precursor, but it is the farnesyl cysteine-carboxyl methyltransferase responsible for the final step in CAAX processing; and so on, but these slips do not detract severely from the overall messages conveyed.

However, as a primer for students, what is most disappointing about this monograph is that it is largely a recitation of facts. In the preface, Madhani is unabashed about his decision to omit all mention of the experiments that led to the level of understanding described in the book. This choice is regrettable because science is a process - a way of thinking and knowing by probing and testing our assumptions about the physical world through experiments. Appreciation for and understanding of the ingenious genetic tactics, incisive biochemical approaches and revealing cell biological methods that were used to tease out the information are the more important lessons to teach. Hence, the dedication to Ira Herskowitz rings a bit hollow because, among his gifts as a scientist, he was a consummate experimentalist. The book is being marketed by CSHL press as an heir to Mark Ptashne's treatise about bacteriophage lambda (A Genetic Switch, now in its 3rd edition). However, Ptashne's book focused rightly on the design and logic of the experiments that led to the conclusions presented. It is ironic that Ptashne was called on to write a brief Forward for Madhani's book, in which he praises the fact that the universal insights gained from studying yeast are presented without describing any of the experiments that led to this knowledge. Nonetheless, as an illustrative guide of how studies in a model organism can be illuminating for large areas of biology, Madhani's book is well worth the price.

\section{COMPETING FINANCIAL INTERESTS}

The author declares that he has no competing financial interests. 